M.G. Burton, R. Jayawardhana \& T.L. Bourke, eds.

\title{
T Tauri Multiple Systems
}

\author{
Alexis Brandeker \\ Stockholm Observatory, AlbaNova University Centre, \\ SE-106 91 Stockholm, Sweden
}

\begin{abstract}
New high-resolution adaptive optics systems provide an unprecedentedly detailed view of nearby star forming regions. In particular, young nearby $\mathrm{T}$ Tauri stars can be probed at much smaller physical scales (a few AU) than possible just a decade ago (several tens of AU). Of major importance is closing the sensitivity gap between imaging and spectral surveys for stellar companions. This allows for 1) calibration of pre-main-sequence evolutionary tracks by obtaining accurate dynamical masses, 2) resolving confusion problems arising by placing unresolved systems in colour-magnitude diagrams, and 3) well defined and determined multiplicity fractions of young stellar systems, important for discriminating star formation scenarios. This article briefly reviews the current status of high resolution imaging of $\mathrm{T}$ Tauri multiple systems, and what we can expect to learn from them in the near future.
\end{abstract}

\section{Introduction}

Most stars, especially pre-main-sequence (PMS) stars, are part of multiple systems (Duchêne 1999; reviews by Tom Greene and Ralf Launhardt, this volume). Since single stars are only in minority, we need to understand how multiple systems are formed in order to understand star formation in general. Because it is currently not possible to deduce a star formation theory from first principles without introducing unrealistic assumptions, observations of young systems are essential for differentiating between possible hypothetical scenarios.

Over the last years, some hundred nearby $\mathrm{T}$ Tauri stars have been imaged with adaptive optics, and before that some hundred were studied by speckle interferometry and lunar occultations. The three examples in Fig. 1 show adaptive optics (AO) imaging of three different multiple $\mathrm{T}$ Tauri stars put to the same spatial scale. They may look surprisingly similar, but are actually typical in two ways: a large fraction of the systems we image are not only binary, but often triple or quadruple, and multiple stars are mostly hierarchically arranged.

Two major questions that can be addressed by high angular resolution observations of multiple stars are:

- How does the early stellar evolution depend on mass (and metallicity)?

- Does the multiplicity frequency for stellar systems evolve in time?

In the following sections I will briefly describe methods of finding answers to these questions, with some emphasis on the contributions from modern $\mathrm{AO}$ 


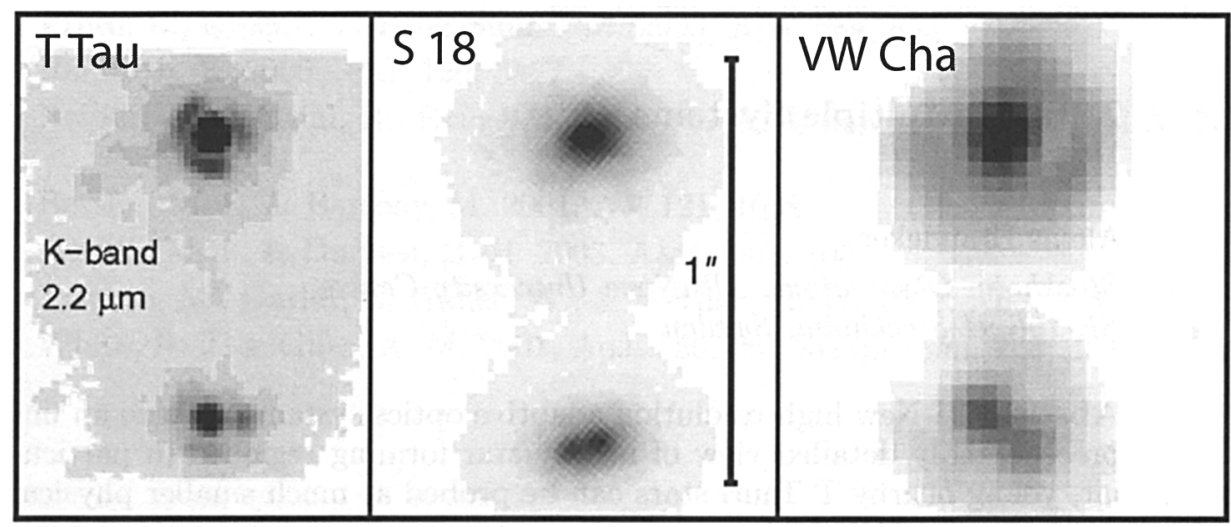

Figure 1. Three different triple $\mathrm{T}$ Tauri systems with a similar appearance: T Tauri, S 18 (in the MBM 12 young star association) and VW Chamaeleontis. The observations, here put to the same angular scale, are from Duchêne, Ghez, \& McCabe (2002), Brandeker, Jayawardhana, \& Najita (2003), and Brandeker et al. (2001) respectively. T Tau was observed in $K$ with Keck AO, S 18 also with Keck AO but in $H$, and VW Cha in $K$ with ADONIS at the $3.6 \mathrm{~m}$ telescope at ESO, La Silla.

systems. I will also review a few representative recent results in the area, and discuss what we may expect in the near future.

\section{Calibrating PMS evolutionary tracks}

Historically, binaries have played a major role in the successful theory of main sequence (MS) stellar structure. The importance of binaries in this context lies in the possibility of direct determination of stellar dynamical masses by following the orbital motion. For PMS stars, a problem has been that the nearest star forming regions are on the order of $100-150 \mathrm{pc}$ distant. With observations limited by a typical seeing disc of $1^{\prime \prime}$, this implies a projected physical resolution of 100-150 AU. Even for massive systems, the orbital period at the resolution separation is expected to be on the order of hundreds to thousands of years, making dynamical mass estimates impractical. Tight short-period binaries may still be found spectroscopically, since spectroscopic searches are limited by flux and not spatial scale. Without spatial information, however, only the relative masses of the stars in a binary can be deduced, unless the inclination of the orbit is known by other means.

\subsection{Dynamical mass determinations}

Clearly observations at higher angular resolutions are essential for dynamical mass determinations. AO systems on modern $8-10 \mathrm{~m}$ facilities regularly achieve diffraction limited imaging in the near infrared (NIR), corresponding to angular resolutions of 30-50 mas. At the distance of recently discovered nearby young 

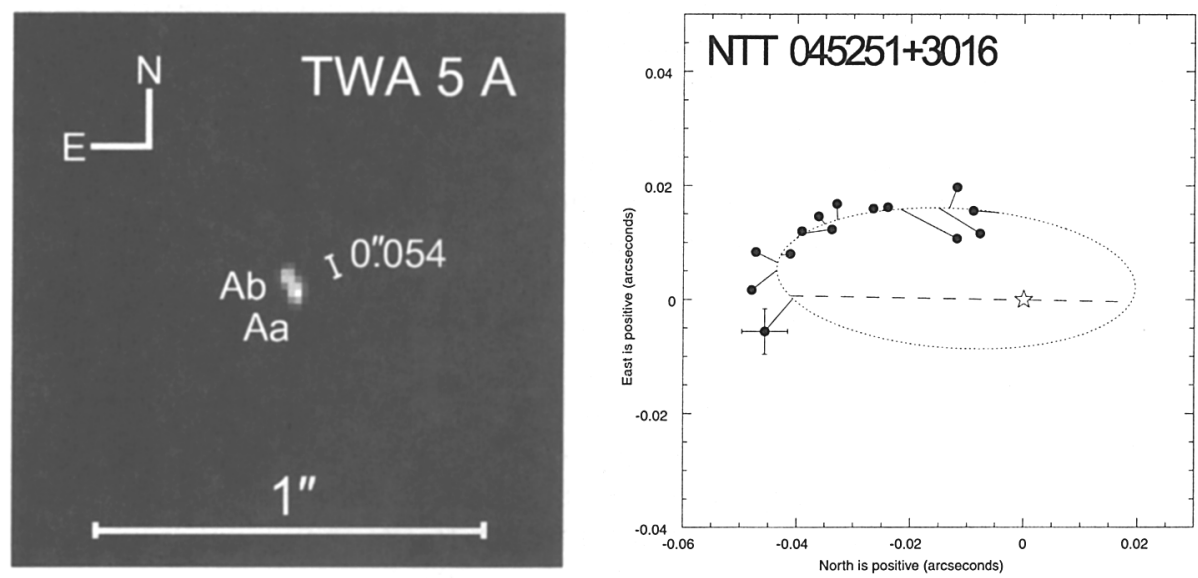

Figure 2. (a, left panel) The tight inner 3 AU binary TWA 5A of the TW Hydrae association. Expected period of binary is 5-10 yr. From Brandeker, Jayawardhana \& Najita (2003). (b, right panel) Orbital solution to PMS binary NTT 045251+3016, making use of both spatially resolved observations and spectroscopic radial velocity measurements. From Steffen et al. (2001).

clusters, like the TW Hydrae association (TWA) at a distance of $\sim 55 \mathrm{pc}$, this translates to a projected physical scale of merely $\sim 2 \mathrm{AU}$. An example of a tight binary close to the resolution limit of the Keck telescope AO is shown in Fig. 2a, displaying TWA 5A with an estimated projected separation of $3 \mathrm{AU}$. By tracking the orbital motion of such tight binaries, it is possible to estimate a dynamical mass within just a few years. NTT $045251+3016$ is a recent example of a binary dynamical mass estimate by Steffen et al. 2001, with their orbital solution shown in Fig. 2b. Using the Fine Guidance Sensor of the Hubble Space Telescope, they were able to reach a relative positional accuracy of 4 mas. Following the orbit during 3 years, a little less than half of the estimated orbital period, and using additional spectroscopically measured radial velocity differences between the two components, individual masses for the stars could be established as $1.45 \pm 0.19 \mathrm{M}_{\odot}$ and $0.81 \pm 0.09 \mathrm{M}_{\odot}$. Possibly surprising, a dynamical distance $d=$ $144.8 \pm 8.3 \mathrm{pc}$ was also derived by combining astrometric and spectroscopic data, independent of any paralactic distance. Schaefer et al. (2003) report three more systems with the system mass dynamically estimated, and show that meaningful dynamical mass estimates can be obtained even though the orbital elements remain very uncertain, as first noted by Eggen (1967).

Other methods that have been employed to determine stellar dynamical masses involve eclipsing binaries (e.g. Covino et al. 2000) and orbital motion of disk gas (Simon, Dutrey, \& Guilloteau 2000). Both have their benefits and caveats: Although eclipsing binaries provide very accurate masses, they require special geometry (very small orbital inclination to the line of sight), and are consequently comparatively rare. By studying disk gas motion also the mass 
of single stars can be dynamically estimated. Gas motion is, however, more sensitive to non-gravitational effects, such as the radiation pressure (see e.g. Olofsson, Liseau, \& Brandeker 2001).

Accurate dynamical mass estimates of young low-mass stars $\left(<1 M_{\odot}\right)$ remain very rare, but given the recent advances in high angular resolution instrumentation, and a few years of orbital motion, the near future will no doubt see the number of dynamically estimated masses multiply.

\subsection{Resolving confusion}

Another problem with seeing limited observations is that of confusion. Tight multiple systems, like those in Fig. 1, are entirely contained in a seeing disc, meaning that photometry will be measured on the system as a whole. Placing the measurements in a Hertzsprung-Russell diagram, the whole system will show an offset from the evolutionary tracks compared to the individual stars. Faint infrared companions close to the primary may show up as an IR-excess in the measured spectral energy distribution, difficult to distinguish from e.g. a warm circumstellar disk. Spectroscopic searches for faint companions are most sensitive to shorter periods, and are inefficient in finding binaries with periods of a couple of years or more. This means physical separations of a few AU or less, translating to some 40 mas or less at the typical distances of 100-150 pc. This is on the same order as the angular resolution of modern AO systems, meaning that there no longer is a sensitivity gap where stellar companions can hide.

\section{Multiplicity frequency evolution}

Most MS stellar systems in the solar neighbourhood are multiple (Duquennoy \& Mayor 1991), and for PMS systems the multiplicity fraction in general seems to be even higher (e.g. Reipurth \& Zinnecker 1993; Duchêne 1999; Barsony, Koresko, \& Matthews 2003). How can this be, if we believe MS field stars to be evolved T Tauri stars? I know of three proposed explanations:

\subsection{Selection effects}

Maybe the multiple fraction excess among PMS stars is due to more sensitive searches (Ghez 1996). For instance, young low-mass companions are brighter and more easily seen than their older counterparts, especially in the NIR where most searches are conducted. However, a more detailed study by White \& Ghez (2001) argues this to not be the case: we do see more companions among young stars, even when looking at the same mass intervals. This point illuminates the importance of finding and correcting observational biases. By closing the sensitivity gap between spectral and spatial searches for companions, corrections for unseen components can be put on a firmer ground.

\subsection{Regional differences}

Maybe different regions produce different multiplicity fractions, and the star forming regions we observe just by chance happen to have higher multiplicity fractions than the average. Indeed, different regions apparently do show different multiplicity fractions (Duchêne 1999). Possible parameters responsible for this 
"environmental effect" are metallicity, volume stellar densities and internal velocity dispersion (see also Woitas, Leinert, \& Köhler 2001; Zinnecker 2003). The statistics are still quite poor, however, so the significance of variations among different regions remains low.

\subsection{Dynamical evolution}

Perhaps multiple systems evolve dynamically; and in the process lose companions, either by stellar mergers or ejections (e.g. Reipurth 2000). One may also speculate that orbital evolution make companions harder to detect. One way is by shrinking a wide, easily resolved, binary to a tight, not so easily resolved binary, another is to widen a moderately wide binary to a very wide (and loose) binary, which will consequently make the companions hard to identify as such (and also make the system more sensitive to disruption due to stellar encounters). By increasing the angular resolution to the point where any stellar companion tighter than the resolution limit would be picked up spectroscopically, the hypothesis of unseen tight binaries can be ruled out. Very wide and loose binaries may be more difficult to find, but can be addressed by multiple epoch wide field studies of proper motions in combination with radial velocity observations, to assess relative space motions.

Detailed numerical studies of the early orbital evolution have produced some quantitative predictions of the low-mass stellar population in young clusters (Bates, Bonnell, \& Bromm 2002, 2003) that can be checked in the near future. Companion ejection requires three-body interaction, and would thus mainly affect the companion fraction defined as $c f=(2 b+3 t+4 q+\ldots) /(s+b+t+q+\ldots)$, where $s, b, t, q$, etc. are the number of single stars, binaries, triples, quadruples etc. The companion fraction is the average number of companions to any primary. The multiplicity fraction $m f=(b+t+q+\ldots) /(s+b+t+q+\ldots)$, on the other hand, should not be as strongly affected since mostly triples or higher order systems eject companions, always leaving a lower order multiple. To disrupt a binary, a stellar encounter is generally needed to provide the third body.

An interesting case where we may be observing a current ejection is the prototype system of all T Tauri stars, T Tauri. Loinard, Rodríguez, \& Rodríguez (2003) used archival Very Large Array (VLA) data to follow the orbital evolution of a centimeter radio emission feature coincident with one of the southern components in the triple system, $\mathrm{T}$ Tau Sb. They derive an orbit of Sb around the unseen (in their data) component Sa, that from 1983 to 1997 was well described by an ellipse, but since then has departed significantly from the initial orbit (Fig. 3a). Furlan et al. (2003) dispute the identification of the radio source with the T Tau Sb companion, using recent AO NIR imaging. By following the evolution of the NIR component since 1997 (when it was first noted in speckle data by Koresko 2000), Furlan et al. (2003) derive a significantly different orbit from that of the radio emission (Fig. 3b). They argue that the centimeter emission probably comes from the ejection of a fourth component in the T Tau system, still unseen in the AO NIR data. Statistically, to observe an ejection in action is very unlikely, so we are extremely fortunate if this is indeed an ejection. Future AO observations in the coming decade will clarify this. 

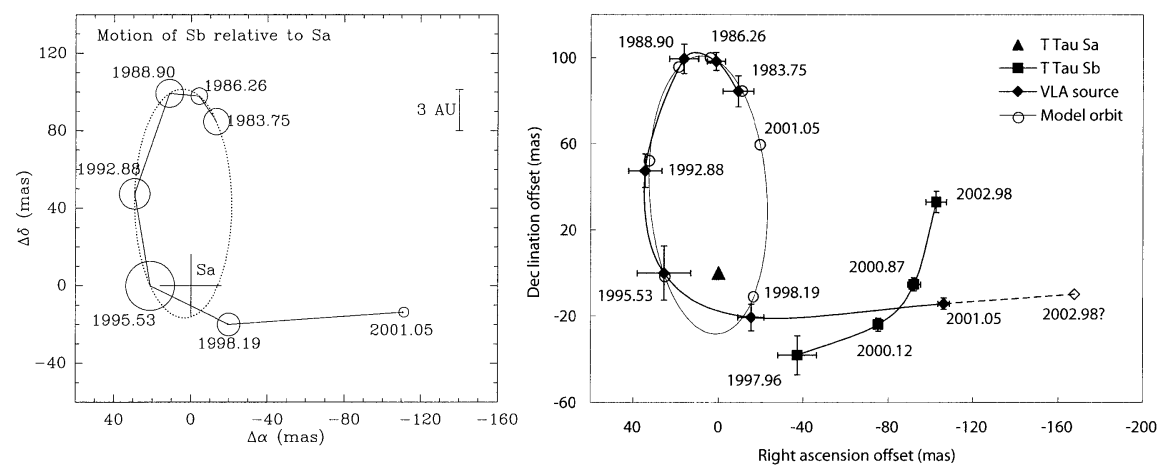

Figure 3. Orbital evolution of the T Tau S system. The left panel shows the centimeter emission feature relative to $\mathrm{T} \mathrm{Tau} \mathrm{Sa,} \mathrm{as} \mathrm{found}$ by VLA (from Loinard, Rodríguez, \& Rodríguez 2003). After 1997, there is an apparent change of orbital path, interpreted as a possible ejection of T Tau Sb. The right panel is from Furlan et al. (2003) and adds recent $\mathrm{AO}$ observations that show this centimeter feature not to be associated with $\mathrm{T}$ Tau $\mathrm{Sb}$, but possibly emanating from a fourth component being ejected.

\section{Summary and outlook}

Adaptive optics systems of today provide, for the first time, high enough angular resolution to determine dynamical masses of the nearest $T$ Tauri multiple systems. A few preliminary estimates of masses have already been reported in the literature.

The coming years will see an increase both in the number of systems with dynamical mass determinations, and in the accuracy of the estimates. Looking beyond the nearest systems, optical interferometry, with notably VLTI and the Keck interferometer, will resolve binaries ten times more distant than AO alone.

By resolving multiple systems, stars can be accurately put on colour-magnitude diagrams and compared to theoretical early evolutionary tracks. In combination with accurate dynamical mass estimates, models will be much better constrained and calibrated for use in, e.g., estimating the initial mass function for different star forming regions.

High angular resolution observations in combination with spectroscopic searches have the potential to carry out a complete census of companions in nearby young clusters. Fig. 4 shows an example of the sensitivity to companions for a state-of-the-art AO system. Note that AO systems now are sensitive enough to probe the "brown dwarf desert" around young stars, as young brown dwarfs are much more luminous than older ones (Baraffe et al. 2003). By increasing the statistics and improving the observational bias corrections, the multiplicity and companion fraction as a function of separation and age may be firmly established, giving important clues to the formation of multiples and stars in general. 


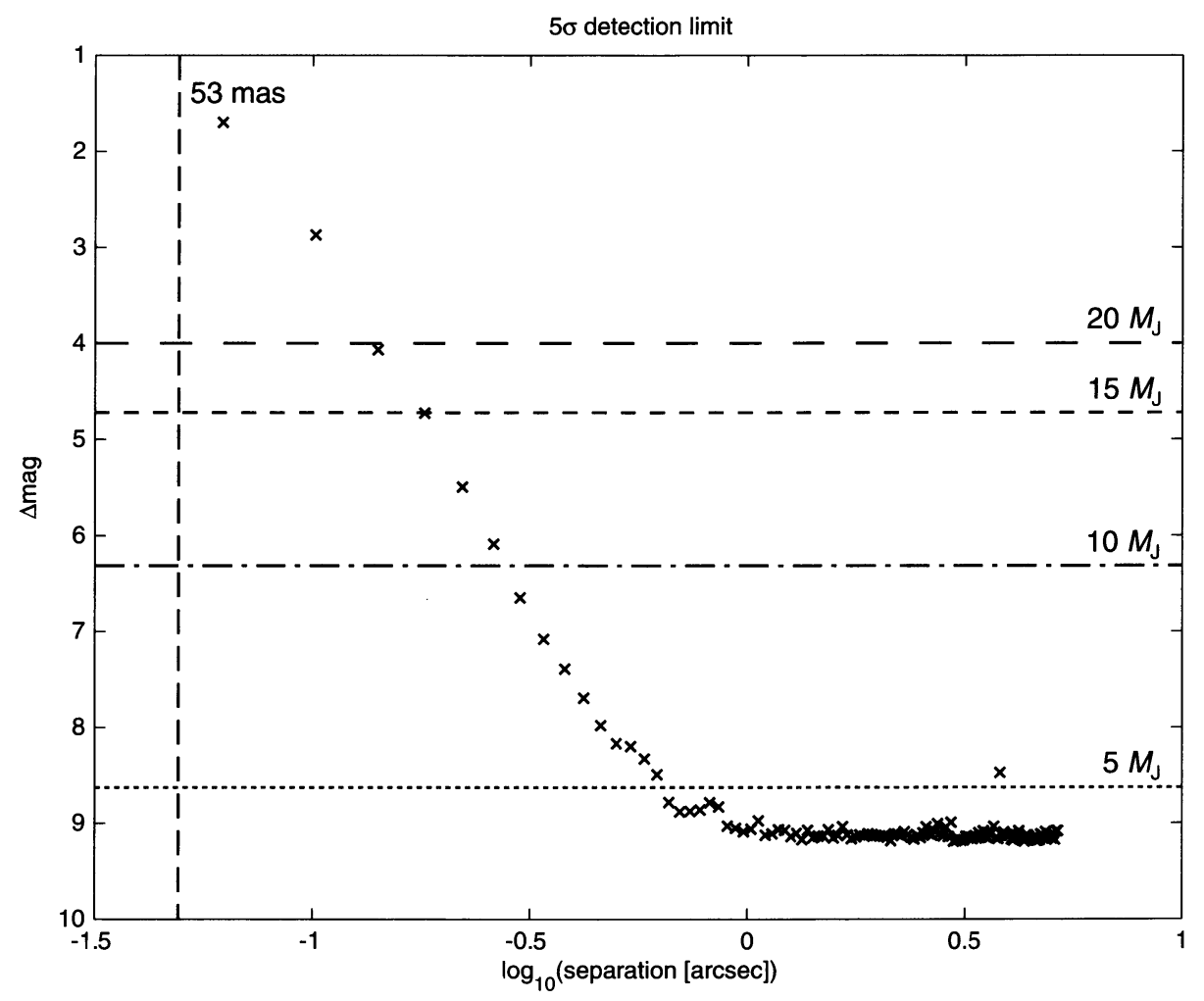

Figure 4. VLT/NACO $5 \sigma$ contrast sensitivity limit in $H$-band as a function of separation for the binary $\eta$ Cha 9 of the $\eta$ Chamaeleontis association (Mamajek, Lawson, \& Feigelson 1999). The vertical dashed line shows the diffraction limit, and the horizontal lines show expected flux ratios of sub-stellar companions at the distance $(\sim 100 \mathrm{pc})$ and magnitude $(H=10)$ of $\eta$ Cha 9 . Luminosities of the sub-stellar companions are derived from Chabrier et al. (2000) and Baraffe et al. (2003), assuming an age of $10 \mathrm{Myr}$. The exposure time was $2190 \times 0.34 \mathrm{~s}$. At small separations the noise is dominated by speckle noise from the point spread function, while the sky-noise and read-out noise dominate at larger separations. From Jayawardhana et al. (2003). 
Acknowledgments. I would like to thank the organisers for inviting me to give this short review, C.F. Liljevalch for a travel grant to get to Sydney, my supervisor René Liseau for supporting my participation despite being close to the deadline of my Ph.D. defence, and Ray Jayawardhana and Gösta Gahm for valuable comments and suggestions.

\section{References}

Barsony, M., Koresko, C., \& Matthews, K. 2003, ApJ, 591, 1064

Bate, M. R., Bonnell, I. A., \& Bromm, V. 2002, MNRAS, 336, 705

Bate, M. R., Bonnell, I. A., \& Bromm, V. 2003, MNRAS, 339, 577

Baraffe, I., Chabrier, G., Barman, T. S., Allard, F., \& Hauschildt, P. H. 2003, A\&A, 402, 701

Brandeker, A., Jayawardhana, R., \& Najita, J. 2003, AJ, 126 (in press)

Brandeker, A., Liseau, R., Artymowicz, P., \& Jayawardhana, R. 2001, ApJ, 561, L199

Chabrier, G., Baraffe, I., Allard, F., \& Hauschildt, P. 2000, ApJ, 542, 464

Covino, E., Catalano, S., Frasca, A., et al. 2000, in IAU Symp. 200, Birth and Evolution of Binary Stars, ed. B. Reipurth \& H. Zinnecker, 9

Duchêne, G. 1999, ApJ, 341, 547

Duchêne, G., Ghez, A. M., \& McCabe, C. 2002, ApJ, 568, 771

Duquennoy, A. \& Mayor, M. 1991, A\&A, 248, 485

Eggen, O. J. 1967, ARA\&A, 5, 105

Furlan, E., Forrest, W. J., Watson, D. M., et al. 2003, submitted to ApJL

Ghez, A. M. 1996, in NATO Advanced Science Institutes Series C Vol. 477, Evolutionary Processes in Binary Stars, ed. R. Wijers \& M. Davies, 1

Jayawardhana, R., Haisch, K., Brandeker, A., \& Mardones, D. 2003, in prep.

Koresko, C. D. 2000, ApJ, 531, L147

Loinard, L., Rodríguez, L. F., \& Rodríguez, M. I. 2003, ApJ, 587, L47

Mamajek, E. E., Lawson, W. A., \& Feigelson, E. D. 1999, ApJ, 516, L77

Olofsson, G., Liseau, R., \& Brandeker, A. 2001, ApJ, 563, L77

Reipurth, B. 2000, ApJ, 120, 3177

Reipurth, B. \& Zinnecker, H. 1993, A\&A, 278, 81

Schaefer, G. H., Simon, M., Nelan, E., \& Holfeltz, S. T. 2003, AJ, in press

Simon, M., Dutrey, A., \& Gulloteu, S. 2000, ApJ, 545, 1034

Steffen, A. T., Mathieu, R. D., Lattanzi, M. G. et al. 2001, ApJ, 122, 997

White, R. J. \& Ghez, A. M. 2001, ApJ, 556, 265

Woitas, J., Leinert, Ch., \& Köhler, R. 2001, A\&A, 276, 982

Zinnecker, H. 2003, in IAU Symp. 212, A Massive Star Odyssey, from Main Sequence to Supernova, ed. K. A. van der Hucht, A. Herrero, \& C. Esteban, in press 